\title{
A Semi-Physiological Population Model to Quantify the Effect of Hematocrit on Everolimus Pharmacokinetics and Pharmacodynamics in Cancer Patients
}

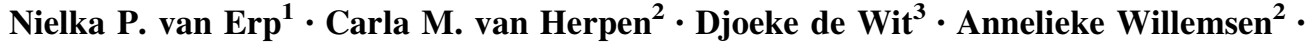 \\ David M. Burger ${ }^{1} \cdot$ Alwin D. R. Huitema ${ }^{4} \cdot$ Ellen Kapiteijn $^{5} \cdot$ Rob ter Heine $^{1}$
}

Published online: 14 June 2016

(c) The Author(s) 2016. This article is published with open access at Springerlink.com

\begin{abstract}
Introduction and Objective Everolimus (a drug from the class of mammalian target of rapamycin [mTOR] inhibitors) is associated with frequent toxicity-related dose reductions. Everolimus accumulates in erythrocytes, but the extent to which hematocrit affects everolimus plasma pharmacokinetics and pharmacodynamics is unknown. We aimed to investigate the everolimus pharmacokinetics/ pharmacodynamics and the influence of hematocrit in cancer patients.

Methods A semi-physiological pharmacokinetic model for everolimus was developed from pharmacokinetic data from 73 patients by non-linear mixed-effects modeling. Using a simulation study with a known pharmacodynamic model describing S6K1 (a downstream mTOR effector) inhibition, we investigated the impact of hematocrit.

Results The apparent volume of distribution of the central and peripheral compartment were estimated to be $207 \mathrm{~L}$ with a relative standard error (RSE) of $5.0 \%$ and $485 \mathrm{~L}$ (RSE $4.2 \%$ ), respectively, with an inter-compartmental clearance of $72.1 \mathrm{~L} / \mathrm{h}$ (RSE $3.2 \%$ ). The apparent intrinsic
\end{abstract}

Rob ter Heine

r.terheine@ radboudumc.nl

1 Department of Pharmacy, Radboud University Medical Center, PO Box 9101, 6500 HB Nijmegen, The Netherlands

2 Department of Medical Oncology, Radboud University Medical Center, Nijmegen, The Netherlands

3 Department of Clinical Pharmacy and Toxicology, Leiden University Medical Centre, Leiden, The Netherlands

4 Department of Pharmacy and Pharmacology, Netherlands Cancer Institute, Amsterdam, The Netherlands

5 Department of Medical Oncology, Leiden University Medical Centre, Leiden, The Netherlands clearance was $198 \mathrm{~L} / \mathrm{h}$ (RSE $4.3 \%$ ). A decrease in hematocrit from $45 \%$ to $20 \%$ resulted in a predicted reduction in whole-blood exposure of $\sim 50 \%$, but everolimus plasma pharmacokinetics and pharmacodynamics were not affected. The predicted S6K1 inhibition was at a plateau level in the approved dose of $10 \mathrm{mg}$ once daily. Conclusions A population pharmacokinetic model was developed for everolimus in cancer patients. Hematocrit influenced whole-blood pharmacokinetics, but not plasma pharmacokinetics or pharmacodynamics. Everolimus whole-blood concentrations should always be corrected for hematocrit. Since predicted mTOR inhibition was at a plateau level in the approved dose, dose reductions may have only a limited impact on mTOR inhibition.

\section{Key Points}

Hematocrit is important for the population wholeblood pharmacokinetics of everolimus, but does not impact plasma pharmacokinetics or mammalian target of rapamycin (mTOR) inhibition.

At the approved dosing regimen, mTOR inhibition (measured as S6K1 inhibition) is at a plateau level.

\section{Introduction}

Everolimus is an orally active inhibitor of the mammalian target of rapamycin (mTOR). Everolimus interacts with FK506 binding protein 1A, $12 \mathrm{kDa}$ (FKBP-12), which results in an inhibitory complex that binds with high affinity to mTOR. Downstream signaling from mTOR 
occurs through an mTOR-Raptor complex, known as TORC1 [1]. The primary downstream targets of mTOR include p70 ribosomal S6 kinase 1 (S6K1) and eukaryotic translation initiation factor $4 \mathrm{E}$ (eIF4E)-binding protein 1 (4EBP1) [2]. The enzyme S6K1 functions in the $\mathrm{G}_{1}$-phase of cell division through phosphorylation of the ribosomal protein S6 to increase the translation of messenger RNA (mRNA) that largely encode ribosomal proteins and other elements of the translational cascade [3]. The phosphorylation of 4EBP1 leads to a reduction of the inhibitory binding to eIF4E. Inhibition of S6K1 in peripheral blood mononuclear cells and skin tissue has been proposed to be an adequate biomarker of mTOR inhibition by everolimus [1].

Despite its proven efficacy, the use of everolimus is seriously hampered by its frequent and severe toxicity. Adverse events that are reported include stomatitis, rash, diarrhea, fatigue, anemia, hyperglycemia, hyperlipidemia, infections, and, less commonly but potentially life threatening, non-infectious pneumonitis [4-6]. In the BOLERO-2 (Breast Cancer Trials of OraL EveROlimus-2) trial, in which breast cancer patients were randomized between everolimus and exemestane versus exemestane, $62 \%$ of the patients treated with the combination required a dose interruption/reduction due to toxicity issues compared with $12 \%$ of the patients treated with exemestane [7]. In the phase III study in patients with metastatic renal cell carcinoma (RECORD-1 [REnal Cell cancer treatment with Oral RAD001 given Daily] study group), $7 \%$ of the patients treated with everolimus required a dose reduction compared with $1 \%$ of the patients treated with placebo, and $38 \%$ needed a dose interruption compared with $11 \%$ treated with placebo [8]. In addition, in patients with advanced pancreatic neuroendocrine tumors (RADIANT-3 [RAD001 in Advanced Neuroendocrine Tumors, Third Trial] study group), $59 \%$ of the patients treated with everolimus required a dose adjustment (reductions or temporary interruptions) compared with $28 \%$ of the patients treated with placebo [6]. This indicates that further research into dose individualization of everolimus is necessary.

Everolimus is rapidly absorbed after oral administration with only a very modest estimated oral bioavailability (5-11\%) and a terminal half-life of approximately $30 \mathrm{~h}$ $[2,9]$. Furthermore, everolimus is metabolized by cytochrome P450 (CYP) isoenzyme 3A4 (CYP3A4), is a substrate for the P-glycoprotein drug transporter, and accumulates in erythrocytes with a fixed erythrocyte to plasma accumulation ratio of $85: 15$ in the clinically relevant concentration range [10]. As a consequence, hematocrit is a known confounder for whole-blood pharmacokinetics, as varying hematocrit will impact the disposition of drugs with a high affinity for red blood cells [11]. This effect is likely to be important with everolimus, as everolimus use leads to anemia in $\sim 16 \%$ of patients [4]. Furthermore, only the unbound plasma concentration of everolimus is able to act on its target. Consequently, for pharmacokinetic and pharmacodynamic analyses, a population pharmacokinetic model describing the everolimus plasma pharmacokinetics, accounting for the effect of hematocrit, is important. However, direct measurement of everolimus plasma concentrations is highly challenging because even minimal hemolysis of everolimus, which accumulates extensively in red blood cells, has a large effect on measured plasma concentrations and everolimus in plasma is not stable $[12,13]$. Therefore, whole-blood concentrations of everolimus are routinely measured in clinical pharmacological studies. Although several models have been published describing the pharmacokinetics of everolimus in whole blood of solid organ transplant patients $[14,15]$, as it stands, no pharmacokinetic model for everolimus is available that accounts for the confounder hematocrit a priori to describe the plasma pharmacokinetics of everolimus in cancer patients. Moreover, unbound everolimus concentrations may be translated to antitumor activity by relating these concentrations to $\mathrm{S} 6 \mathrm{~K} 1$ inhibition [16]. Our purpose was, therefore, to develop a population pharmacokinetic model in cancer patients treated with everolimus and to investigate the impact of varying hematocrit on the pharmacokinetics and in silico pharmacodynamics of everolimus.

\section{Methods}

\subsection{Study Participants and Pharmacokinetic Sampling}

A total of 73 patients from a phase II study investigating the efficacy of everolimus for the treatment of progressive unresectable recurrent or metastatic thyroid cancer (THYRRAD; $n=41$ ) (ClinicalTrials.gov identifier NCT01118065) and a phase IV study investigating the influence of age and body weight on everolimus disposition in patients with metastatic breast cancer (INPRES [Influence of Exceptional Patient Characteristics on Everolimus Exposure]; $n=32$ ) (ClinicalTrials.gov identifier NCT01948960) were included in the present analysis. Patients were treated continuously with an oral dose of everolimus $10 \mathrm{mg}$ once daily until tumor progression, unacceptable toxicity, or death. Concurrent use of drugs recognized as being strong inhibitors or inducers of the isoenzyme CYP3A during treatment or within the 5 days prior to enrollment was prohibited. Adequate renal function (Modification of Diet in Renal Disease [MDRD] $>30 \mathrm{~mL} /$ min) and hepatic function (AST or ALT $<5 \times$ upper limit of normal) was required. The studies were approved by the 
institutional medical ethics committees of the Leiden University Medical Center and Radboud university medical center. All patients gave written informed consent for participation in these studies.

\subsection{Bioanalysis}

Whole-blood EDTA samples were obtained at days 1 and 15 of therapy in the THYRRAD study and at days 14 and 35 in the INPRES study. In the THYRRAD study either limited sampling $(0,1,2$, and $3 \mathrm{~h}$ after drug intake) or extensive sampling $(0,1,2,3,4,5,6,7$, and $8 \mathrm{~h})$ was performed. In the INPRES study only extensive pharmacokinetic sampling, as described for the THYRRAD study, was performed. Everolimus concentrations in EDTA whole blood were measured using independently validated assays: an ultra-performance liquid chromatography-tandem mass spectrometric (UPLC-MS/MS) assay was used in the THYRRAD study and a validated high-performance liquid chromatography-tandem mass spectrometric (HPLC-MS/ MS) assay in the INPRES study. Validation of both assays was performed according to the European Medicines Agency (EMA) guidelines for bioanalytical method development [17]. The UPLC-MS/MS could quantify everolimus concentrations over the range of $2-160 \mu \mathrm{g} / \mathrm{L}$. The HPLC-MS/MS method could quantify everolimus in whole blood over the range of $1-150 \mu \mathrm{g} / \mathrm{L}$. To assure comparable results, the performance of both assays was tested in each analytical run by incorporating the same external quality controls of Recipe ${ }^{\circledR}$ (RECIPE Chemicals + Instruments $\mathrm{GmbH}$, Munich, Germany), which met the acceptance criteria $(<15 \%$ bias) for all analyses.

\subsection{Pharmacokinetic Analysis}

Pharmacokinetic analysis of the observed whole-blood pharmacokinetics was performed by means of non-linear mixed effects modelling with the software program NONMEM $^{\circledR}$ (version 7.30; Icon Development Solutions, Ellicott City, MD, USA), using Piraña ${ }^{\circledR} 2.9 .2$ as an interface for Perl Speaks NONMEM (PsN; version 4.4.8) and R statistics (version 3.2.0) [18]. The first-order estimation method with interaction (FOCE-I) in NONMEM $^{\circledR}$ was used throughout the analysis. Precision of parameter estimates was calculated using the covariance step in NONMEM $^{\circledR}$. For hierarchical models, a $p$ value $<0.01$, corresponding to a drop in the objective function of $>6.63$ units per parameter, was considered statistically significant. Throughout model building, we used stringent criteria to prevent ill-conditioning of the model: a successful covariance step, a condition number $<1000$, and parameter correlations $<0.95$ were prerequisites throughout model building. Also, basic goodness-of-fit plots and visual predictive checks of the model and observed whole-blood pharmacokinetics were generated and inspected. As an internal validation, a bootstrap analysis $(n=500)$ was performed of the final model.

\subsection{Structural Pharmacokinetic Model}

The pharmacokinetic parameters in the model were estimated on the available whole-blood concentrations, and the model predicted the corresponding plasma pharmacokinetic parameters from paired whole blood and hematocrit level observations based on the information discussed below.

From the US Food and Drug Administration (FDA) New Drug Application reviews of everolimus for solid organ transplant and cancer patients, it is known that the erythrocyte:plasma concentration ratio of everolimus is constant at a fixed ratio of 85:15 in the clinical concentration range of $5-100 \mu \mathrm{g} / \mathrm{L}[10,13]$. Therefore, whole-blood concentrations can be described using the following equation (Eq. 1):

$C_{\mathrm{wb}}=C_{\mathrm{p}} \times(1+4.667 \times \mathrm{Ht})$

where $C_{\mathrm{wb}}$ is the whole-blood concentration, $C_{\mathrm{p}}$ is the plasma concentration, and $\mathrm{Ht}$ is hematocrit. This was implemented in our structural pharmacokinetic model, similar to that recently described by Størset et al. [11] for the immunosuppressant drug tacrolimus. In the model, from the paired observations of whole-blood concentrations and hematocrit levels, plasma concentrations are calculated with Eq. (1) in the \$ERROR block of the NONMEM $^{\circledR}$ control stream. Consequently, all parameter estimates are expressed as plasma pharmacokinetic parameters and the model is capable of predicting both whole-blood and plasma concentrations, provided that a hematocrit level is known for each observation.

Furthermore, the unbound everolimus (plasma) concentrations $\left(C_{\mathrm{fu}}\right)$ that drove the pharmacodynamic model were derived from total everolimus plasma concentrations with the knowledge that a fraction of 0.27 , irrespective of hepatic function or hematocrit level, is unbound in plasma $\left(C_{\mathrm{fu}}=\right.$ $\left.C_{\mathrm{p}} \times 0.27\right)$, with only a limited variability of $3 \%[10,12]$.

Absorption was described with a chain of transition compartments. The mean absorption time (MAT) was estimated and the rate constant for these transition compartments was calculated using Eq. (2):

$k_{\mathrm{tr}}=\frac{n+1}{\mathrm{MAT}}$

where $k_{\text {tr }}$ is the transit rate constant and $n$ equals the number of transition compartments, as described previously $[19,20]$. The relationship between presystemic and systemic hepatic metabolism was accounted for by implementing a physiological well-stirred liver 
model, as previously proposed by Gordi et al. [21]. In this model, the apparent intrinsic hepatic clearance $\left(\mathrm{CL}_{\mathrm{int}} / F\right)$ for everolimus was estimated, assuming a liver blood flow $\left(Q_{\mathrm{H}}\right)$ of $90 \mathrm{~L} / \mathrm{h}$ and a hepatic volume $\left(V_{\text {liver }}\right)$ of $1 \mathrm{~L}$. The liver plasma flow $\left(Q_{\mathrm{HP}}\right)$ was calculated using the following formula (Eq. 3):

$Q_{\mathrm{HP}}=Q_{\mathrm{H}} \times(1-\mathrm{Ht})$

The hepatic extraction $\left(E_{\mathrm{H}}\right)$ was defined as Eq. (4):

$E_{\mathrm{H}}=\frac{\mathrm{CL}_{\mathrm{int}} \times f_{\mathrm{u}}}{Q_{\mathrm{HP}}+\left(\mathrm{CL}_{\mathrm{int}} \times f_{\mathrm{u}}\right)}$

where $f_{\mathrm{u}}$ represents the unbound everolimus fraction of 0.27 in plasma [10]. Hepatic plasma clearance $\left(\mathrm{CL}_{\mathrm{H}}\right)$ was calculated using the following formula (Eq. 5):

$\mathrm{CL}_{\mathrm{H}}=E_{\mathrm{H}} \times Q_{\mathrm{HP}}$

A schematic depiction of our pharmacokinetic model is shown in Fig. 1. Furthermore, inter-individual and interoccasion variability were assumed to be log-normally distributed.

The rate constants describing the pharmacokinetic model (see Fig. 1) were as follows:

$$
\begin{aligned}
& k_{\text {tr }}=\frac{n+1}{\mathrm{MAT}} \\
& k_{20}=\frac{\mathrm{CL}_{\mathrm{H}}}{V_{\text {liver }}} \\
& k_{23}=\frac{Q_{\mathrm{HP}} \times\left(1-E_{\mathrm{H}}\right)}{V_{\text {liver }}} \\
& k_{32}=\frac{Q_{\mathrm{HP}}}{V_{3}} \\
& k_{34}=\frac{Q}{V_{3}}
\end{aligned}
$$

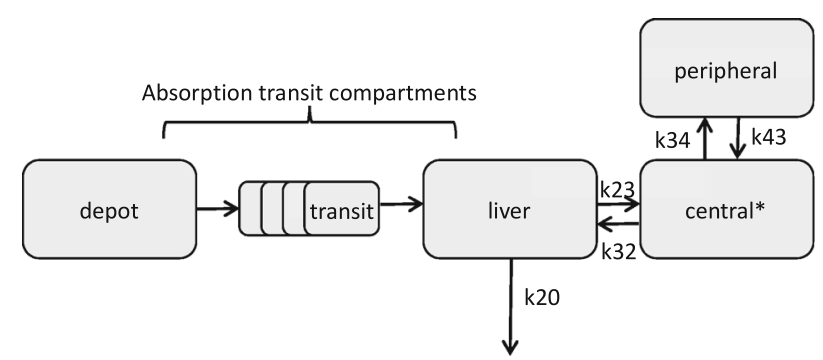

* Plasma concentrations are calculated from the paired observations of whole blood concentrations and hematocrit, assuming the known 85:15 red-blood-cell-to-plasma accumulation ratio of everolimus

Fig. 1 Pharmacokinetic model. Asterisk indicates plasma concentrations are calculated from the paired observations of whole-blood concentrations and hematocrit, assuming the known 85:15 red blood cell:plasma accumulation ratio of everolimus
$k_{43}=\frac{Q}{V_{4}}$

\subsection{Predicted Effect of Unbound Everolimus Plasma Concentrations on S6K1 Inhibition}

For the simulation study, a previously developed pharmacokinetic/pharmacodynamic model directly relating inhibition S6K1 with unbound plasma concentrations of everolimus was implemented (Eq. 6) [16]:

S6K1 inhibition $(\%)=\frac{I_{\max } \times C_{\mathrm{u}}}{\mathrm{IC}_{50}+C_{\mathrm{u}}}$

where $I_{\max }, \mathrm{IC}_{50}$, and $C_{\mathrm{u}}$ are the maximum percentage inhibition of S6K1 activity (97\%) in tumor tissue, the unbound everolimus plasma concentration that inhibits $50 \%$ of S6K1 activity $(0.05 \mathrm{ng} / \mathrm{mL})$, and the unbound everolimus plasma concentration, respectively [16, 22].

It should be noted that in our simulation study, S6K1 inhibition was not measured in cancer patients but predicted from the individually predicted unbound plasma concentrations from our pharmacokinetic model as an input for the pharmacodynamic model. For the simulation studies, the typical population whole-blood and plasma concentrations as well as S6K1 inhibition were simulated during a $24 \mathrm{~h}$ dosing interval at steady state for the $10 \mathrm{mg}$ once-daily dosing regimen, the approved starting dose of everolimus. As the pharmacokinetics and pharmacodynamics are influenced by hematocrit, the everolimus pharmacokinetics and pharmacodynamics were simulated at hematocrit levels of $20 \%$ and $45 \%$ of red blood cells in total blood volume, representing the typical lower and upper values of hematocrit that can be found in cancer patients [23]. Lastly, we simulated the steady-state trough (pre-dose) whole-blood and plasma concentrations and the corresponding S6K1 inhibition for the $10 \mathrm{mg}$ once-daily dosing regimens at hematocrit levels of 20 and $45 \%$ for 1000 patients on two occasions (2000 observations for each hematocrit and dose level). Trough concentrations were considered most relevant since S6K1 inhibition is directly related to everolimus plasma concentrations, which are lowest just before intake of a new dose.

\section{Results}

\subsection{Study Participants}

The patient and study characteristics are summarized in Table 1. As shown, a large range in hematocrit was observed among the participants. A total of 915 blood samples were collected in these 73 patients. 
Table 1 Patient and study characteristics

\begin{tabular}{lcll}
\hline Characteristics & $n$ & Median & Range \\
\hline $\begin{array}{l}\text { Number of study participants } \\
\text { Age (years) }\end{array}$ & & 62 & $37-80$ \\
Sex $(n)$ & 22 & & \\
$\quad$ Male & 51 & & \\
$\quad$ Female & & 73.2 & $45-105$ \\
Weight (kg) & & 10 & $10-15$ \\
Everolimus dose per occasion (mg) & & 38 & $25-49.7$ \\
Hematocrit (\%) & 947 & & \\
Number of whole-blood concentrations & 947 & \\
\hline
\end{tabular}

\subsection{Estimation Results}

The parameter estimates for the developed pharmacokinetic model for everolimus, presented as the corresponding plasma pharmacokinetic parameters, are shown in Table 2. A two-compartment first-order pharmacokinetic model including implementation of a well-stirred liver model described the pharmacokinetics well. Oral absorption was best described with a total of four transit compartments and a MAT of $0.544 \mathrm{~h}$. As shown in Table 2, the apparent volume of distribution of the central and peripheral compartment were estimated to be 207 and $485 \mathrm{~L}$, respectively, with an inter-compartmental clearance of $72.1 \mathrm{~L} / \mathrm{h}$. The apparent intrinsic clearance was estimated to be $198 \mathrm{~L} / \mathrm{h}$.
These parameters could be reliably estimated, as shown by their low relative standard errors (RSE $<6.4 \%$ ).

The inter-individual variability in MAT, intrinsic clearance, and volume of distribution of the central compartment were estimated to be $62.0,38.9$, and $36.1 \%$, respectively. Although the physiologically plausible correlation between intrinsic clearance and volume of distribution of the central compartment could not be reliably estimated (a high RSE of 90.6\%), it significantly $(p<0.001)$ improved the model and was therefore included in the model. Also, inter-occasion variability on relative bioavailability (F1) was introduced in the model and this significantly improved the model fit $(p<0.001)$ and was estimated to be $15.2 \%$. Introduction of two separate residual error models for the two studies did not improve the model.

The parameter estimates of the final model were close to bootstrap averages and the parameters could be precisely estimated (see Table 2). The basic goodness-of-fit plots of the model are depicted in Fig. 2 and did not show substantial bias. Figure $2 \mathrm{e}$ shows the conditional weighted residuals versus hematocrit and a uniform distribution of weighted residuals was observed, indicating that our assumed relationship between hematocrit, plasma concentrations, and whole-blood concentrations provided unbiased predictions. Figure 3 shows the prediction-corrected visual predictive check, based on 1000 simulations of the

Table 2 Model parameters

\begin{tabular}{|c|c|c|c|c|}
\hline \multirow[t]{2}{*}{ Model parameter ${ }^{\mathrm{a}}$} & \multicolumn{2}{|c|}{ Final model parameter estimates } & \multicolumn{2}{|c|}{$\begin{array}{l}\text { Bootstrap results } \\
(n=500)\end{array}$} \\
\hline & Estimate & RSE (CV \%) & Mean & RSE (CV \%) \\
\hline MAT through 4 transit compartments (h) & 0.544 & 6.4 & 0.549 & 8.4 \\
\hline $\mathrm{CL}_{\text {int }} / F(\mathrm{~L} / \mathrm{h})$ & 198 & 4.3 & 198 & 4.7 \\
\hline$V_{3} / F(\mathrm{~L})$ & 207 & 5.0 & 207 & 6.1 \\
\hline$Q(\mathrm{~L} / \mathrm{h})$ & 72.1 & 3.2 & 72.9 & 5.0 \\
\hline$V_{4} / F(\mathrm{~L})$ & 485 & 4.2 & 489 & 5.6 \\
\hline Inter-individual variability of MAT (\%) & 62.0 & 23.5 & 61.8 & 21.7 \\
\hline Inter-individual variability of intrinsic clearance (\%) & 38.9 & 24.8 & 37.0 & 22.5 \\
\hline Inter-individual variability of $V_{3} / F(\%)$ & 36.1 & 63.4 & 35.9 & 71.8 \\
\hline Correlation between inter-individual variability of intrinsic clearance and $V_{3} / F$ & 0.347 & 90.6 & 0.292 & 98.0 \\
\hline Inter-occasion variability on F1 (\%) & 15.2 & 43.1 & 15.8 & 32.5 \\
\hline Red blood cell:plasma concentration ratio & \multicolumn{4}{|l|}{$85: 15$ (fixed) } \\
\hline Unbound fraction in plasma & \multicolumn{4}{|l|}{0.27 (fixed) } \\
\hline Inter-individual variability in unbound fraction (\%) & \multicolumn{4}{|l|}{3 (fixed) } \\
\hline Residual error (\%) & 26.0 & 20.8 & 25.9 & 18.2 \\
\hline Condition number & \multicolumn{4}{|l|}{64.32} \\
\hline
\end{tabular}

$C L_{i n t} / F$ intrinsic clearance, $C V$ coefficient of variation, $F 1$ absorbed fraction, $M A T$ mean absorption time, $Q$ inter-compartmental clearance, $R S E$ relative standard error, $V_{3} / F$ volume of the central compartment, $V_{4} / F$ volume of the peripheral compartment

a All pharmacokinetic parameters are shown as the corresponding plasma pharmacokinetic parameters 
A

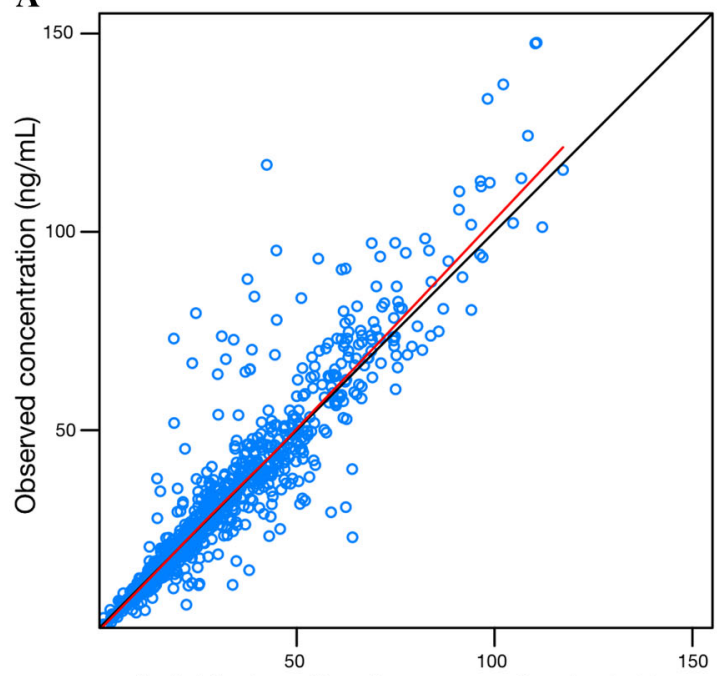

Individual predicted concentration (ng/mL)

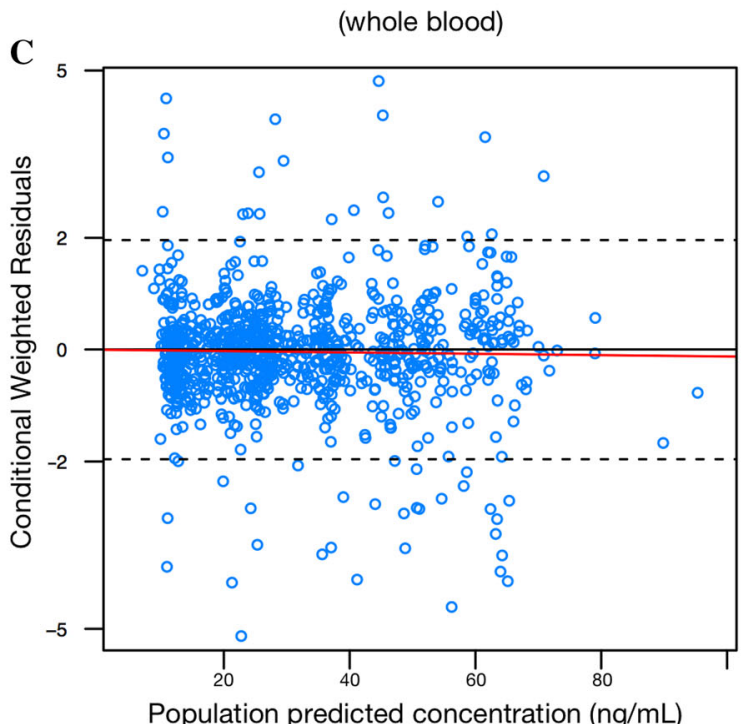

Population predicted concentration $(\mathrm{ng} / \mathrm{mL})$

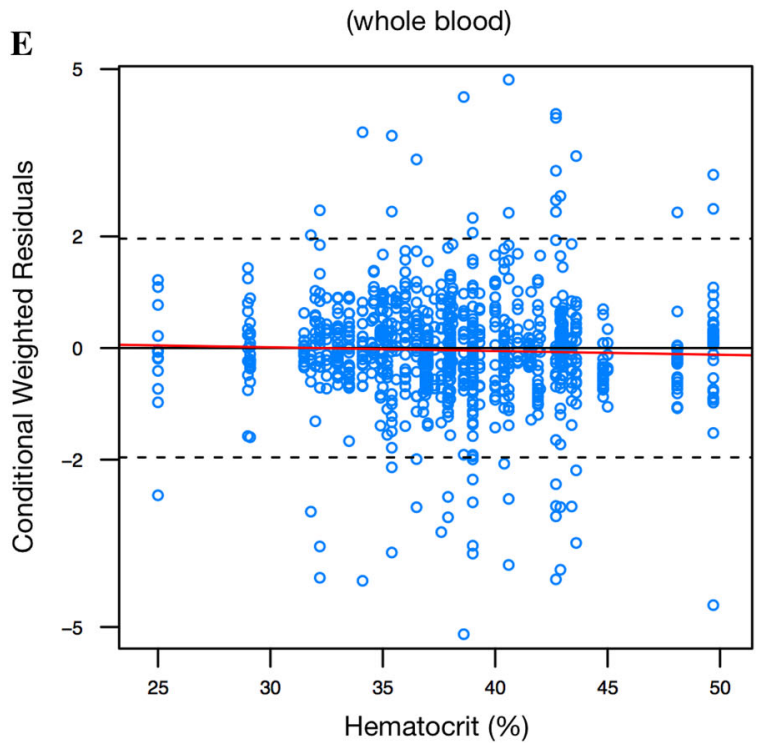

B

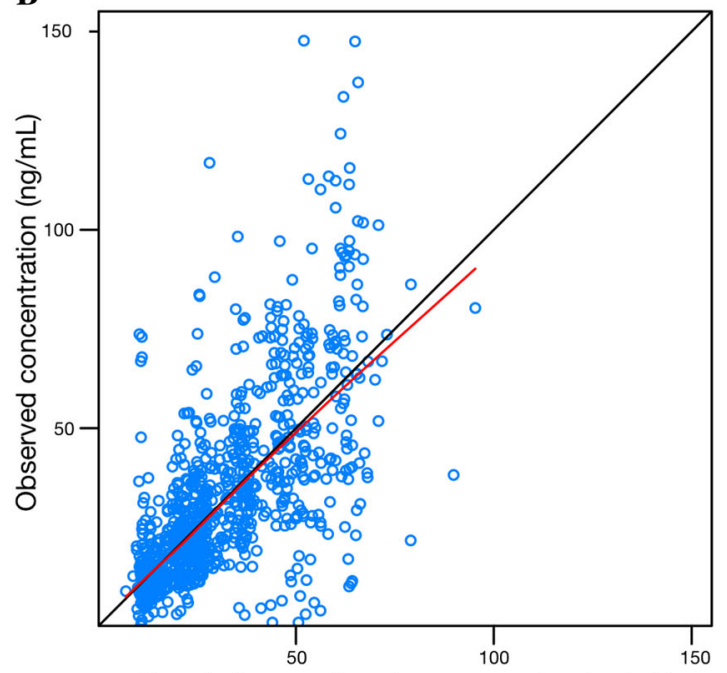

Population predicted concentration $(\mathrm{ng} / \mathrm{mL})$

(whole blood)

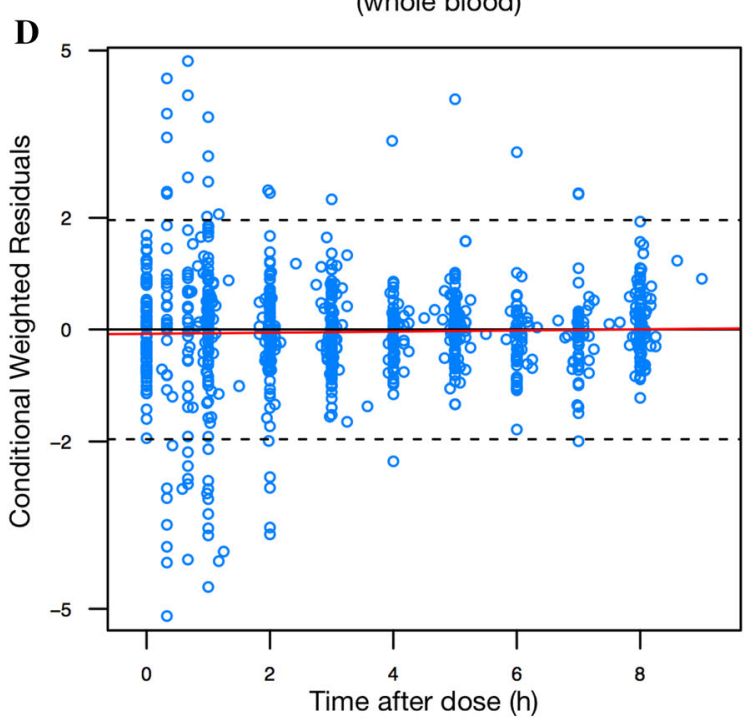


४Fig. 2 Goodness-of-fit plots: a observed concentration vs. individual predicted concentration (whole blood); b observed concentration vs. population predicted concentration (whole blood); c conditional weighted residuals vs. population predicted concentration (whole blood); d conditional weighted residuals vs. time after dose; e conditional weighted residuals vs. hematocrit

original dataset. As observed in this figure, the simulated data correspond well with the observed data.

\subsection{Pharmacokinetic and Pharmacodynamic Simulation Results}

The population-predicted whole-blood and plasma concentrations and corresponding S6K1 inhibition at steady state during a $24 \mathrm{~h}$ dosing interval with an everolimus $10 \mathrm{mg}$ once-daily dose for a typical patient with a 20 and $45 \%$ hematocrit level are shown in Fig. 4. As shown, hematocrit impacted the whole-blood pharmacokinetics of everolimus but did not influence its plasma pharmacokinetics and, therefore, S6K1 inhibition (Table 3). Furthermore, it can be observed that, despite the high predicted variability in trough whole-blood and plasma concentrations, as reflected in their wide $90 \%$ prediction intervals, the variability in the corresponding S6K1 inhibition was limited. The simulated steady-state trough (pre-dose) whole-blood concentrations (Table 3) were below the required trough concentration to inhibit S6K1 adequately in patients with a hematocrit of 0.2 and above this concentration in patients with a hematocrit of 0.45. However, since the corresponding plasma concentrations and thereby the unbound everolimus concentrations are nearly equal, these concentration differences will theoretically not result in differences in efficacy.

\section{Discussion and Conclusion}

We have successfully developed a semi-physiological population pharmacokinetic model for everolimus in patients with cancer. The model reliably captured all observed whole-blood pharmacokinetic data and could be used to predict plasma concentrations as well as mTOR (S6K1) inhibition when the hematocrit level is known.

We observed two interesting findings in our study. Firstly, our analysis clearly demonstrates that hematocrit relevantly impacts whole-blood concentrations while plasma concentrations remain more or less the same. In patients with cancer, large variation in hematocrit is commonly observed [23, 24]. This will result in variable wholeblood concentrations. Since only the unbound everolimus plasma concentration is responsible for the pharmacological effect of everolimus, measured whole-blood concentrations should always be corrected for hematocrit, to interpret the relationship between everolimus exposure and treatment outcome, which is currently not done in practice $[25,26]$. Secondly, we showed that despite high variability in systemic everolimus exposure among patients, throughout a dosing interval the model-predicted mTOR (S6K1) inhibition was nearly complete and that despite high variability in pharmacokinetics, variability in S6K1 inhibition was only modest. This indicated that at the current dosing regimen the mTOR inhibition may be at the top end of the concentration-effect curve and that dose reductions may not necessarily result in less mTOR
Fig. 3 Visual predictive check for the final pharmacokinetic model of everolimus, based on $n=500$ simulations.

Prediction-corrected simulated (shaded areas) and observed (circles and lines) everolimus whole-blood concentrations versus time after dose (h). The thick red line connects the observed median values per bin. The dotted red lines connect the 5th and 95th percentiles of the observations. The blue areas are the $95 \%$ confidence interval of the 5th and 95th percentiles. The red area indicates the confidence interval of the median

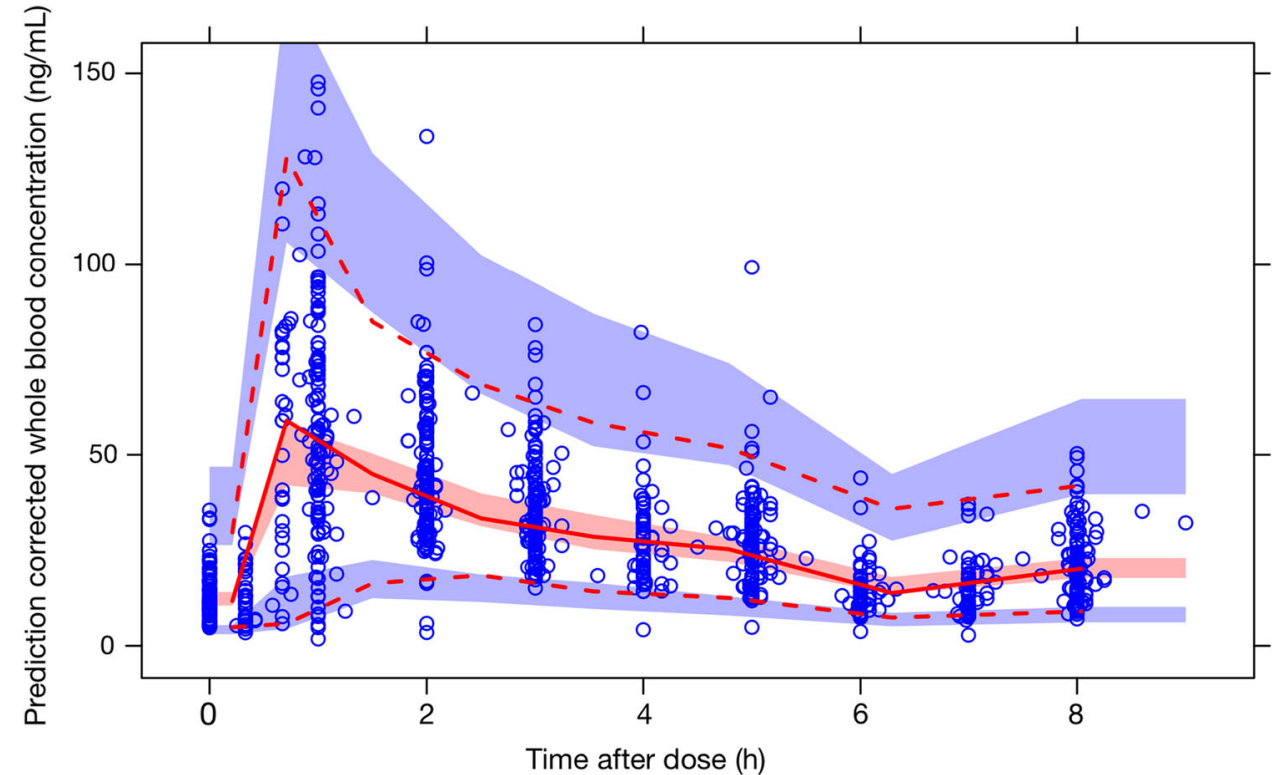


A Whole blood concentrations versus time

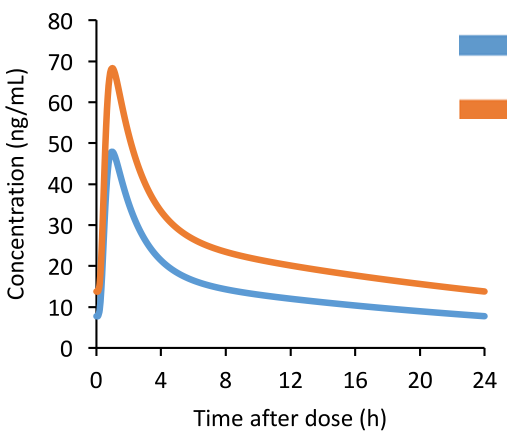

B Plasma concentrations versus time

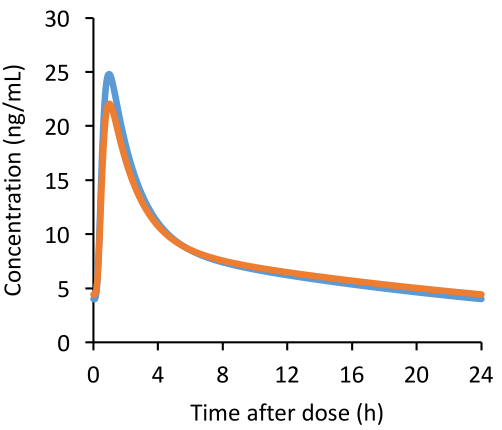

C Tumor S6K1 inhibition versus time

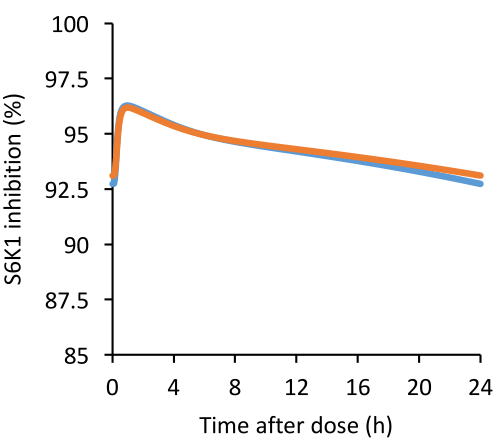

Fig. 4 Population predicted pharmacokinetics and pharmacodynamics of everolimus at steady state: a whole-blood concentrations versus time; b plasma concentrations versus time; c tumor S6K1 inhibition versus time. $Q D$ once daily inhibition. This encourages further prospective in vivo investigation to reduce everolimus toxicity without loss of efficacy.

Although recently several population pharmacokinetic models for everolimus have been described, we believe our model adds to the knowledge currently available because the previously described models studied a different patient population (solid organ transplant patients) and used very different dose levels with an empirical pharmacokinetic model instead of a semi-physiologically well-stirred liver model without accounting for hematocrit and erythrocyte accumulation [14, 27, 28]. Furthermore, in contrast with previous work, our model enables prediction of the plasma concentration and this allows everolimus plasma pharmacokinetics to be linked with its pharmacodynamics.

Our pharmacokinetic model relied on assumptions of erythrocyte and plasma protein binding of everolimus, based on the best available data, which could not be verified in vivo. All results of the simulation study should be interpreted with this in mind. One may argue that the absence of measured (unbound) plasma concentrations is a shortcoming of our study. As stated in the Introduction, quantification of everolimus plasma concentrations may be challenging, and the protein binding is known to be concentration independent with very limited variability $[10,12,13]$. Although we accounted for the limited variability in plasma protein binding in our simulation study, we could not account for variability in erythrocyte binding, as this is unknown. Thus, the variability in plasma concentrations and S6K1 inhibition in our simulation study, as presented in Table 3, may be under-predicted. It should also be noted that saturation of everolimus accumulation may occur at concentrations higher than usually observed in routine clinical practice, and that the fixed accumulation ratio might not be applicable in this situation. Prediction of everolimus pharmacokinetics with our model at substantially higher concentrations than usually observed when using $10 \mathrm{mg}$ once daily should, therefore, be performed

Table 3 Predicted trough concentrations and S6K1 inhibition at 20 and $45 \%$ hematocrit

\begin{tabular}{|c|c|c|c|c|c|c|}
\hline \multirow[t]{3}{*}{ Trough concentration } & \multicolumn{6}{|l|}{ Hematocrit } \\
\hline & \multicolumn{3}{|l|}{$20 \%$} & \multicolumn{3}{|l|}{$45 \%$} \\
\hline & $C_{\mathrm{wb}}(\mathrm{ng} / \mathrm{L})$ & $C_{\mathrm{pl}}(\mathrm{ng} / \mathrm{L})$ & S6K1 inhibition (\%) & $C_{\mathrm{wb}}(\mathrm{ng} / \mathrm{L})$ & $C_{\mathrm{pl}}(\mathrm{ng} / \mathrm{L})$ & S6K1 inhibition (\%) \\
\hline Median & 7.85 & 4.06 & 92.8 & 14.0 & 4.51 & 93.2 \\
\hline $90 \%$ PI & $3.31-18.7$ & $1.71-9.65$ & $87.5-95.2$ & $6.19-30.6$ & $2.00-9.86$ & $88.9-95.2$ \\
\hline
\end{tabular}

Simulations were based on 1000 virtual individuals with observations on two separate occasions

$C_{p l}$ everolimus concentration in plasma, $C_{w b}$ everolimus concentration in whole blood, $P I$ prediction interval 
with caution. Future studies should be directed towards establishing everolimus erythrocyte-binding constants and its associated variability in order to account for this variability and possible saturated binding at higher wholeblood concentrations. Furthermore, red blood cell binding of a drug is, in rare cases, known to be influenced by other drugs [29]. This may cause a clinically relevant pharmacokinetic interaction for drugs that extensively accumulate in erythrocytes, such as everolimus. As it stands, there are no data supporting displacement of everolimus from erythrocytes, but since this may relevantly change its plasma pharmacokinetics, this warrants further research.

The pharmacodynamic model describing the relationship between unbound plasma concentrations and S6K1 inhibition used may not representative of the human situation, as it was initially developed in rodents. However, there has been extensive research on this subject and this showed that differences in pharmacokinetics in rodents and humans were the only determinants for observed differences in S6K1 inhibition and that there was only a limited difference between tumor-bearing rats and cancer patients regarding the concentration effect of everolimus and its effect on signal transduction proteins such as S6K1 [22]. Therefore, this pharmacokinetic/pharmacodynamic model was also used to select the everolimus doses of 5 and $10 \mathrm{mg}$ for the clinical phase II and III trials for treatment of solid malignancies [16]. Consequently, we think the model used is adequate to predict S6K1 inhibition, but it encourages prospective in vivo evaluation.

In addition, the use of strong inhibitors or inducers of CYP3A, the main enzyme involved in everolimus metabolism [10], and impairment of gastrointestinal function or gastrointestinal disease that may significantly alter the absorption of study drugs were exclusion criteria in our study. Therefore, extrapolation from our pharmacokinetic model to these situations may be limited. Finally, extrapolation of our model to different dosing regimens should be performed with caution since dose non-proportional pharmacokinetics cannot be ruled out over the large dosing range used within oncology. Currently, there are no data that support the assumption of non-linear pharmacokinetics; however, this should be investigated before broader use of this model.

For our simulation study, we implemented a previously developed pharmacokinetic/pharmacodynamic model that describes the relationship between unbound plasma concentrations of everolimus and tumor S6K1 inhibition and this model was used to rationally guide clinical development of everolimus dosing schedules. The variability in predicted tumor S6K1 inhibition in our study may be under-estimated, as this previously developed model does not account for variability in the pharmacodynamics parameters. Also, this pharmacodynamic model only accounted for tumor S6K1 inhibition. As it stands, no pharmacokinetic/pharmacodynamic model is available to describe the relationship between plasma concentrations and inhibition of peIF-4G, another downstream effector of mTOR. It is known, however, that a higher drug exposure is necessary for complete inhibition of other downstream mTOR pathways than S6K1. The clinical relevance of this difference remains unknown and should be further investigated. However, as S6K1 inhibition is considered a good biomarker for monitoring mTOR inhibition [16, 22, 30], it would be interesting and relevant to investigate the effect of variable plasma everolimus concentrations on S6K1 inhibition in patients treated with everolimus and correlate the S6K1 inhibition potential to treatment outcome. This will help to better understand the mechanism underlying everolimus-induced efficacy and toxicity and the involvement of everolimus pharmacokinetics herein [7].

The current analysis clearly demonstrates that hematocrit relevantly influences whole-blood concentrations while plasma concentrations remain unaffected. Since the unbound everolimus concentration is available to interact with the target, we believe that plasma concentrations should be used to investigate exposure-treatment outcome relationships. Our semi-physiological model can be used for this purpose.

\section{Compliance with Ethical Standards}

Acknowledgments The authors would like to acknowledge Elisabet Størset at the Oslo University Hospital in Norway for her helpful discussions on NONMEM $^{\circledR}$ control stream coding for erythrocyte accumulation of everolimus. Jan den Hartigh from the Leiden University Medical Center and Karin Hoogtanders from the Maastricht University Medical Center are also greatly acknowledged for their help in bioanalysis of everolimus. Novartis is acknowledged for financially funding the clinical study with everolimus.

Conflict of interest N. P. van Erp has received a research grant from Novartis to perform the presented study with everolimus. C. M. van Herpen, D. de Wit, A. E. C. A. B. Willemsen, D. M. Burger, A. D. R. Huitema, E. Kapiteijn, and R. ter Heine declare that they have no conflicts of interest.

Ethical Approval All procedures performed in studies involving human participants were in accordance with the ethical standards of the institutional and/or national research committee and with the 1964 Helsinki Declaration and its later amendments or comparable ethical standards.

Informed consent Informed consent was obtained from all individual participants included in the study.

Open Access This article is distributed under the terms of the Creative Commons Attribution-NonCommercial 4.0 International License (http://creativecommons.org/licenses/by-nc/4.0/), which permits any noncommercial use, distribution, and reproduction in any medium, provided you give appropriate credit to the original author(s) and the source, provide a link to the Creative Commons license, and indicate if changes were made. 


\section{References}

1. O'Reilly T, McSheehy PM. Biomarker development for the clinical activity of the mTOR inhibitor everolimus (RAD001): processes, limitations, and further proposals. Transl Oncol. 2010;3(2):65-79.

2. O'Donnell A, Faivre S, Burris HA III, Rea D, Papadimitrakopoulou V, Shand N, et al. Phase I pharmacokinetic and pharmacodynamic study of the oral mammalian target of rapamycin inhibitor everolimus in patients with advanced solid tumors. J Clin Oncol. 2008;26(10):1588-95.

3. Jastrzebski K, Hannan KM, Tchoubrieva EB, Hannan RD, Pearson RB. Coordinate regulation of ribosome biogenesis and function by the ribosomal protein S6 kinase, a key mediator of mTOR function. Growth Factors. 2007;25(4):209-26.

4. Baselga J, Campone M, Piccart M, Burris HA 3rd, Rugo HS, Sahmoud T, et al. Everolimus in postmenopausal hormone-receptor-positive advanced breast cancer. $N$ Engl J Med. 2012;366(6):520-9.

5. Motzer RJ, Escudier B, Oudard S, Hutson TE, Porta C, Bracarda $S$, et al. Efficacy of everolimus in advanced renal cell carcinoma: a double-blind, randomised, placebo-controlled phase III trial. Lancet. 2008;372(9637):449-56.

6. Yao JC, Shah MH, Ito T, Bohas CL, Wolin EM, Van Cutsem E, et al. Everolimus for advanced pancreatic neuroendocrine tumors. N Engl J Med. 2011;364(6):514-23.

7. Rugo HS, Pritchard KI, Gnant M, Noguchi S, Piccart M, Hortobagyi $\mathrm{G}$, et al. Incidence and time course of everolimus-related adverse events in postmenopausal women with hormone receptor-positive advanced breast cancer: insights from BOLERO-2. Ann Oncol. 2014;25(4):808-15.

8. Motzer RJ, Escudier B, Oudard S, Hutson TE, Porta C, Bracarda $\mathrm{S}$, et al. Phase 3 trial of everolimus for metastatic renal cell carcinoma : final results and analysis of prognostic factors. Cancer. 2010;116(18):4256-65.

9. EMA. Affinitor-EPAR public assessment report. 29 May 2009. http://www.ema.europa.eu/docs/en_GB/document_library/EPAR_-Public_assessment_report/human/001038/WC500022817.pdf. Accessed 1 Dec 2015.

10. US FDA. Afinitor - clinical pharmacology and biopharmaceutics review(s). 2008. http://www.accessdata.fda.gov/drugsatfda_docs/ nda/2009/022334s000_ClinPharmR.pdf. Accessed 6 Apr 2016.

11. Størset E, Holford N, Hennig S, Bergmann TK, Bergan S, Bremer $\mathrm{S}$, et al. Improved prediction of tacrolimus concentrations early after kidney transplantation using theory-based pharmacokinetic modelling. Br J Clin Pharmacol. 2014;78(3):509-23.

12. Kovarik JM, Sabia HD, Figueiredo J, Zimmermann H, Reynolds $\mathrm{C}$, Dilzer SC, et al. Influence of hepatic impairment on everolimus pharmacoltinetics: Implications for dose adjustment. Clin Pharmacol Ther. 2001;70:425-30.

13. US FDA. Certican — clinical pharmacology and biopharmaceutics review(s). http://www.fda.gov/ohrms/dockets/ac/05/briefing/ 2005-4183B1_02_08-FDA-Clin-Pharm-Original.pdf. Accessed 6 Apr 2016.

14. Moes DJA, Press RR, den Hartigh J, van der Straaten T, de Fijter JW, Guchelaar H-J. Population pharmacokinetics and pharmacogenetics of everolimus in renal transplant patients. Clin Pharmacokinet. 2012;51(7):467-80.

15. Felipe C, Oliveira N, Hannun P, de Paula MI, Tedesco-Silva H, Medina-Pestana JO. Pharmacokinetics and long-term safety and tolerability of everolimus in renal transplant recipients converted from cyclosporine. Ther Drug Monit. 2016;38(1):64-72.
16. Tanaka C, O'Reilly T, Kovarik JM, Shand N, Hazell K, Judson I, et al. Identifying optimal biologic doses of everolimus (RAD001) in patients with cancer based on the modeling of preclinical and clinical pharmacokinetic and pharmacodynamic data. J Clin Oncol. 2008;26(10):1596-602.

17. EMA. Guideline on bioanalytical method validation. 21 July 2011. http://www.ema.europa.eu/docs/en_GB/document_library/ Scientific_guideline/2011/08/WC500109686.pdf. Accessed 1 Dec 2014.

18. Keizer RJ, Karlsson M, Hooker A. Modeling and simulation workbench for NONMEM: tutorial on Pirana, PsN, and Xpose. CPT Pharmacomet Syst Pharmacol. 2013;2(6):e50.

19. ter Heine R, Scherpbier HJ, Crommentuyn K, Bekker V, Beijnen $\mathrm{JH}$, Kuijpers TW, et al. A pharmacokinetic and pharmacogenetic study of efavirenz in children: dosing guidelines can result in subtherapeutic concentrations. Antivir Ther. 2008;13(6):779-87.

20. Rousseau A, Leger F, Le Meur Y, Saint-Marcoux F, Paintaud G, Buchler M, et al. Population pharmacokinetic modeling of oral cyclosporin using NONMEM: comparison of absorption pharmacokinetic models and design of a Bayesian estimator. Ther Drug Monit. 2004;26(1):23-30.

21. Gordi T, Xie R, Huong NV, Huong DX, Karlsson MO, Ashton M. A semiphysiological pharmacokinetic model for artemisinin in healthy subjects incorporating autoinduction of metabolism and saturable first-pass hepatic extraction. Br J Clin Pharmacol. 2005;59(2):189-98.

22. O'Reilly T, McSheehy PMJ. Biomarker development for the clinical activity of the mTOR inhibitor everolimus (RAD001): processes, limitations, and further proposals. Transl Oncol. 2010;3(2):65-79.

23. Knight K, Wade S, Balducci L. Prevalence and outcomes of anemia in cancer: a systematic review of the literature. Am J Med. 2004;116(7):11-26.

24. Hinderling PH. Red blood cells: a neglected compartment in pharmacokinetics and pharmacodynamics. Pharmacol Rev. 1997;49(3):279-95.

25. Ravaud A, Urva SR, Grosch K, Cheung WK, Anak O, Sellami DB. Relationship between everolimus exposure and safety and efficacy: meta-analysis of clinical trials in oncology. Eur J Cancer. 2014;50(3):486-95.

26. Thiery-Vuillemin A, Mouillet G, Nguyen Tan Hon T, Montcuquet $\mathrm{P}$, Maurina T, Almotlak H, et al. Impact of everolimus blood concentration on its anti-cancer activity in patients with metastatic renal cell carcinoma. Cancer Chemother Pharmacol. 2014;73(5):999-1007.

27. Lemaitre F, Bezian E, Goldwirt L, Fernandez C, Farinotti R, Varnous S, et al. Population pharmacokinetics of everolimus in cardiac recipients: comedications, ABCB1, and CYP3A5 polymorphisms. Ther Drug Monit. 2012;34(6):686-94.

28. Kovarik JM, Hsu CH, McMahon L, Berthier S, Rordorf C. Population pharmacokinetics of everolimus in de novo renal transplant patients: impact of ethnicity and comedications. Clin Pharmacol Ther. 2001;70(3):247-54.

29. Lettieri JT, Portelli ST. Effects of competitive red blood cell binding and reduced hematocrit on the blood and plasma levels of [14C] Indapamide in the rat. J Pharmacol Exp Ther. 1983;224(2):269-72.

30. Tabernero J, Rojo F, Calvo E, Burris H, Judson I, Hazell K, et al. Dose- and schedule-dependent inhibition of the mammalian target of rapamycin pathway with everolimus: a phase I tumor pharmacodynamic study in patients with advanced solid tumors. J Clin Oncol. 2008;26(10):1603-10. 